the gelatin, and then a slow diffusion of the remaining salt takes place downwards, and this might give rise to the idea that the thing was really growing.

Woodbridge School, Suffolk. W. A. Douglas Rudge.

\section{The Spectrum of the Positive Rays (Canal-Strahlen).}

IN former publications (Ann. d. Phys., vol. xiv., p. 524, I904; vol. xvi., p. 490, 1905; “ Die Elektrizität in Gasen," Leipzig, 1902, pp. 446, 457) I have expressed the opinion that the carrier of the line spectrum of a chemical element is the positive atom-ion, while, on the other hand, the band spectrum is due to the re-combination of positive atom-ions with negative electrons. From this it would follow that the particles of the positive rays (being positive atom-ions) should emit the line spectrum of the gas in which they are produced. Moreover, since these particles possess a considerable velocity, their spectrum lines, observed in the direction of the rays, should have a position and breadth differing from the position and breadth when the lines are observed in a direction normal to the rays. Again, since the positive rays ionise the gas traversed by them, re-combination of positive and negative ions must take place in their path; the gas traversed by the positive rays must therefore emit the band spectrum, which will be superposed on the line spectrum due to the positive rays themselves. The lines of the band spectrum must have the same position whether they are observed in the direction of the positive rays or in a direction at right angles to them, inasmuch as their carriers do not possess the velocity of the positive rays.

I now state briefly the results of an experimental spectrographic examination of the light emitted by a gas traversed by positive rays. (I) Nitrogen shows simultaneously the band and the line spectrum, hydrogen shows simultaneously the series spectrum $(\mathrm{H} \alpha, \mathrm{H} \beta, .$.$) and the many lines$ spectrum. (2) The line spectrum emitted normally to the positive rays and the line spectrum emitted in the direction of the rays are different; the former shows in hydrogen sharp lines of the known wave-length, while the latter shows these "stationary" lines, and besides them, on their ultra-violet side, new widened lines ("displaced" lines). (3) This displacement is greater when the velocity of the positive rays is greater. (4) The lines of the band spectrum (many lines spectrum) have the same position and breadth whether they are observed in the direction of the positive rays or in a direction at right angles to them. A full account of the investigation will be published shortly.

Göttingen, November 3 .

J. STARK.

\section{Replicas of Diffraction Gratings.}

KINDLY allow me to correct a statement contained in your notice of Mr. R. J. Wallace's replicas of diffraction gratings in your issue of November 2 (vol. 1xxiii. p. 21).

It is there stated, as also in the Astrophysical Journal from which the extract was taken, that I first flood the grating with oil in my method of producing replicas. This I may say I have never done except when making experiments, my procedure being exactly the same as $\mathrm{Mr}$. Wallace's, viz. to flood the grating direct with the clarified celluloid solution, dry it in much the same way, but using special precautions to ensure perfectly even drying, stripping and mounting in a similar manner to $\mathrm{Mr}$. Wallace, but leaving out the gelatin coating, which in my opinion is quite unnecessary.

I beg to enclose you one of Mr. Wallace's first quality (average) replicas, kindly sent to me by him in exchange for one of my own, as also a couple of mine for comparison. The great difference to be noted in their surfaces and performance is due to the peculiarities in the surfaces of the original gratings, one of my own replicas having a brightness in the first spectrum on one side of at least four times that of the other, and twice that of Mr. Wallace's replica.

The grating from which the very bright replica is taken is a "Rowland" of 14,438 lines to the inch, and was formerly the property of the late Dr. Common. The original of the other is a very beautiful specimen of recent work on the Rowland engine, 15,038 lines to the inch. Now, whilst the latter when mounted on parallel plane glass gives comparatively feeble spectra, when mounted on prisms for direct-vision purposes, and tilting the prism to the angle required for the minimum deviation for the diffraction spectrum, first order, the brightness approaches that from the "Common" grating, whilst its much greater freedom from scattered light renders it very suitable for prominence and similar work, the dispersion being about equal to five $60^{\circ}$ flint glass prisms in the centre of the spectrum, and decidedly greater at the red end.

This increase of brightness is, of course, attributable to the form of the grooves, less interference being produced under the latter condition, and this notwithstanding the increase in dispersion.

It may be of interest to some to know that I have succeeded in mounting these grating films on a perfectly flattened ring of glass, so that, by avoiding the use of glass as a base, light of very short wave-length can be examined by this means, either in the one case by transmission to about $\lambda 2600$ or by reflection to as low as $\lambda \quad 1850$, and possibly lower. (The discovery of this ieflective property for ultra-violet light was made by $\mathrm{Mr}$. Morris-Airey, of the Victoria University, last year.) In order to examine by reflection either a partial vacuum is created behind the film when mounted on a glass ring or the film is mounted on a concave surface, which, although not giving the lines of the grating their true form, gives very fair resolution.

1 have also succeeded in making conceve replicas practically as perfect as plane ones, by rotating the grating during the drying process at such a rate that the paraboloidal curvature of the solution was practically the same as that of the grating. Anyway, the difference is so slight that when dry no rings can be seen on examining it by monochromatic light before the film is stripped from the grating. The difficulty of silvering these replicas satisfactorily has, however, prevented further progress, for the present at least.

In justice to Mr. Wallace I ought to say that, in a reply to a letter from me, he states he obtained his information from a patent I once took out in connection with the application of these grating replicas to colour photography; but the method there described is not the one I have adopted in making my replicas.

Whitefield, near Manchester, November 6.

The article referred to by Mr. Thorp was, as mentioned in its first two lines, simply a résumé of Mr. Wallace's article in the Astrophysical Journal; and the statement corrected by $\mathrm{Mr}$. Thorp was taken from that journal. It may be said, however, that the three gratings sent by Mr. Thorp have been compared, under similar conditions, and the results are in full accordance with the descriptions given above.

The second "Thorp" replica (I5, I 20 lines) is a beautiful specimen in appearance, having none of the mottling which appears on the 14.438 -line copy, but its first-order spectra are excelled in brightness by the first-order spectrum on one side of the latter, which is a similar replica to those that have produced such remarkably good results in eclipse and other observations when attached to just an ordinary hand-camera or a simple modification thereof.

The Writer of the NOTICE.

\section{Aurora of Novemb:r 15 .}

AN extraordinary aurora was seen here on November ${ }^{15}$ at 6 p.m., in appearance something like a "stormy" sunset. The lower part of the aurora was illumined with a bluish-green light, and had an altitude at the centre of about $10^{\circ}$. Above this, extending for a further ten or fifteen degrees, the sky was brilliantly illuminated with streamers of a rosy red colour. I did not wait for its disappearance, but at $9.3^{\circ} \mathrm{p} . \mathrm{m}$. red streamers were visible in the N.N.W.

I looked for the Leonids this morning continuously from 1.20 to 2.35 from the vantage ground of the Dartmoor hills ( ${ }_{5} 500$ feet). I saw only one Leonid at 2.30 , but the moon would prevent small ones being seen. The night was perfectly clear.

Buckfastleigh, S. Devon, November 16. 